\section{Neue Antiinfektiva und Impfstoffe}

\author{
N. Suttorp ${ }^{1}$ \\ ${ }^{1}$ Medizinischen Klinik m. S. Infektiologie und Pneumologie der \\ Charité
}

Die Lebenserwartung in der westlichen Welt hat sich in den letzten 150 Jahren nahezu verdoppelt. Hygienemaßnahmen, Vakzinierung und Antiinfektiva - die Einführung dieser drei Prinzipien haben (neben ausreichender und besserer Ernährung) wesentlich beigetragen, dieses wirklich erstaunliche Ergebnis zu erzielen.

1936 wurden Sulfonamide zugelassen. Dann folgten ca. 20 weitere Antibiotika-Klassen (ca. 80 verschiedene Antibiotika). Es geht um Penicilline, Aminoglykoside, Tetrazykline, Makrolide, Cephalosporine, Glykopeptide, Fluorchinolone, Carbapeneme, Oxazolidinone und verschiedene $\beta$-Laktamase-Inhibitoren. In den 1980er- und 1990er-Jahren erfolgten zahlreiche Markteinführungen, im ersten Jahrzehnt des neuen Jahrhunderts gab es jedoch ein großes Tief. Seit 2010 wurden wieder etwas mehr Antibiotika zugelassen [1]. Bei vielen Neuzulassungen handelt es sich jedoch um altbekannte Klassen und veränderte Moleküle bekannter Grundstrukturen wie Glycopeptide oder Oxazolidinone. Jetzt und in $\mathrm{Zu}$ kunft werden auch vermehrt Kombinationen von einem alten Cephalosporin und einem neuen Beta-Laktamase-Inhibitor oder einem neuen Cephalosporin und einem alten Beta-Laktamase-Inhibitor zur Vergügung stehen.

Mit der Einführung des INH in den 50er Jahren und dann mit der Zulassung von Ethambutol, Rifampicin und Pyrazinamid in den 60er Jahren wurde Tbc eine einigermaßen behandelbare Krankheit. 1995 kam Rifabutin, eine Rifampicin-Modifikation, dazu. Mehr als 40 Jahre hat es gedauert, bis wirklich neuartige TBc-Medikamente kamen: 2013 das Bedaquilin und 2014 das Delamanid. Angesichts der MDR- und der XDR-Problematik gibt es jetzt wenigstens zwei dringlich erwünschte Ergänzungen.

Auch wurden in den letzten 50 Jahren mehr als 30 neue antivirale Medikamente entwickelt. Das reicht von $A=$ Abacavir (Ziagen) bis Z = Zanamivir (Relenza).

Nystatin, Amphotericin B und Griseofulvin waren die ersten zugelassenen Antimykotika. Dann folgten verschiedene Azole und dann die Echinocandine. Zuletzt wurde in 2015 das Isavuconazol verfügbar. Insgesamt stehen wirksame Therapien bei Pilzinfektionen von Candida bis hin zur Mukormykose zur Verfügung.

Seit den 60er Jahren wurden > 20 neue Impfstoffe zugelassen. Das betrifft Mumps, Masern, Röteln, Windpocken, Hepatitis B, Meningokokken, Hämophilus, Hepatitis A, Papillomviren. Ich wurde in den 60er Jahren noch gegen Pocken geimpft. Die Impfung gegen Pocken war so erfolgreich, dass sie vor > 30 Jahren ausgesetzt werden konnte.
Hepatitis C ist auf einmal heilbar geworden, HIV-Patienten sterben nicht mehr an, sondern mit HIV. Die Pocken sind ausgerottet. So kann die Infektionsmedizin in den letzten 50 Jahren zahlreiche Erfolge vorzeigen.

In Verbindung mit Hygienemaßnahmen haben Antiinfektiva und Vakzine- wie erwähnt- entscheidend dazu beigetragen, die Lebenserwartung zu verdoppeln. Daraus wird unmittelbar verständlich, was auf dem Spiel steht, wenn an den drei Fronten Schwächen erkennbar werden. Und die Gefahren sind nicht gering: Hygiene-Maßnahmen werden nicht immer konsequent eingehalten. Impfmüdigkeit nimmt zu, es gibt einen harten Kern an Impfgegnern und Antibiotika- bzw. Antiinfektiva-Resistenzen nehmen zu.

Kürzlich wurden Berechnungen aus England bekannt, nach denen sich im Jahre 2050 die Zahl der Todesfälle durch multiresistente Keime weltweit von 700000 auf 10 Millionen erhöhen wird (in Europa von 23000 auf 400000), wenn nicht neue Antibiotika entwickelt werden [2]. Auch die Behandelbarkeit der Tuberkulose hat sich durch MDR- und XDRMykobakterien verschlechtert. Das Problem der Antibiotikaresistenz ist in der Politik angekommen und war 2015 beim G7-Gipfel ein wichtiges Thema.

Es gilt, in den nächsten Dekaden den Erfolg von Antiinfektiva zu stabilisieren. Wir müssen auf einen vorsichtigen, adäquaten Einsatz von Antibiotika drängen, und wir müssen verstärkt Therapien erforschen, die über Antibiotika hinausgehen (adjunktive Therapie). Wir benötigen aber auch immer wieder neue Antibiotika. Denn Antibiotikaresistenzen gehören zur normalen Ökologie, und sie werden uns somit immer begleiten.

Die Zukunft der Vakzinierung in den nächsten Dekaden kann man optimistisch denken. Der methodologische Fortschritt ist enorm (reverse vaccinology 2.0, synthetische Vakzine (z. B. automatisierte Glycansynthese)). Weitere Vakzine gegen Pathogene und Pathogenprodukte werden möglich sein. Noch mehr Engagement in der Erforschung von Vakzinen ist auch deshalb eine sinnvolle zukünftige Ausrichtung, da diese keinen Resistenzdruck ausüben.

Hohe Aufmerksamkeit in der Weiterentwicklung von Antiinfektiva und Vakzine ergibt sich zudem aus der Erfahrung der vergangenen Jahrzehnte, dass jährlich einige neue Erreger bekannt werden, die neu sind oder neu entdeckt wurden. Als wenige Beispiele seien hier pars pro toto Legionellen, Hantaviren, Borrelien, HIV, Helicobacter, Hepatitis C-Viren, SARS- und MERS-Corona-Viren genannt.

Interessenkonflikte: Keine

\footnotetext{
Literatur

1 Im Internet: http://www.vfa.de/de/arzneimittel-forschung/woranwir-forschen/neue-antibiotika-den-vorsprung-wahren.html; Stand: 06.09.2016

2 O'Neill J. The review on antimicrobial resistance, May 2016. Im Internet: https://amr-review.org/sites/default/files/160525_Final\%20paper_with\%20cover.pdf; Stand: 06.09.2016
} 


\section{Bibliografie}

DOI http://dx.doi.org/10.1055/s-0042-114457

Drug Res 2016; 66, Suppl. 1: S11-S12

(c) Georg Thieme Verlag KG Stuttgart · New York . ISSN 2194-9379
Korrespondenzadresse

Prof. Dr. Norbert Suttorp

Direktor der Medizinischen Klinik

m.S. Infektiologie und Pneumologie

Charité Universitätsmedizin Berlin

Charitéplatz 1

10117 Berlin

norbert.suttorp@charite.de

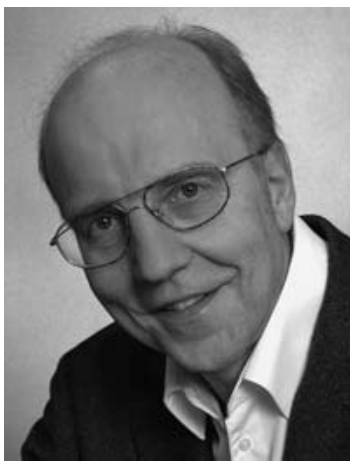

